\title{
A Comparative Assessment of Presence of Biological Debris And Level of Contamination on Rotary And Hand Endodontic Files Subjected To Different Cleaning Protocols Prior of Sterilization- A Stereomicroscope Study
}

\author{
Setu R. Bavaria ${ }^{1}$, Nimisha C. Shah ${ }^{2,}$ Ruchirani P. Shah ${ }^{3}$, Deep J. Makati ${ }^{4}$, \\ Dhaval V. Bhadra ${ }^{5}$

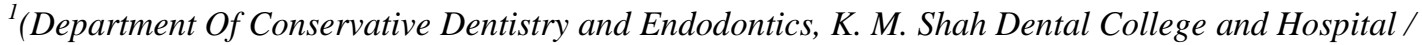 \\ Sumandeep Vidyapeeth, India) \\ ${ }_{2}^{2}$ (Department Of Conservative Dentistry and Endodontics, K. M. Shah Dental College and Hospital / \\ Sumandeep Vidyapeeth, India) \\ ${ }^{3}$ (Department Of Conservative Dentistry and Endodontics, K. M. Shah Dental College and Hospital / \\ Sumandeep Vidyapeeth, India) \\ ${ }^{4}$ (Department Of Conservative Dentistry and Endodontics, K. M. Shah Dental College and Hospital $/$ \\ Sumandeep Vidyapeeth, India) \\ ${ }^{5}$ (Department Of Conservative Dentistry and Endodontics, K. M. Shah Dental College and Hospital / \\ Sumandeep Vidyapeeth, India)
}

\begin{abstract}
To assess the presence of biological debris and level of contamination on rotary and hand endodontic files subjected to different cleaning protocols prior to sterilization using a stereomicroscope. Ninety endodontic instruments (hand and rotary file systems), after clinical use in the Department of Conservative Dentistry and Endodontics, KMSDCH, Piparia, Vadodara were used for the study. The instruments were brushed manually with a nylon brush for 10 strokes and randomly divided into five groups (3\% hydrogen peroxide, $2 \%$ glutaraldehyde solution, $0.2 \%$ chlorhexidine gluconate, $3 \%$ sodium hypochlorite, ultrasonic bath with an enzymatic solution). Fifteen files directly from the manufacturer's packaging were used as control. All the instruments in the experimental groups were finally rinsed in distilled water for five minutes and dried. All the instruments were stained for 3 minutes, rinsed, dried and visualized under the stereomicroscope for presence of residual organic debris at 40X magnification. Statistical analysis was done by Kruskal-Wallis Test and Mann Whitney Test. Control group showed highest percentage of debris collection, ( $p$ value $=\langle 0.001)$ whereas among experimental groups there was no statistically significant difference but group 5showed less debris among all other groups. Mechanical cleaning combined with ultrasonic bath showed excellent debris elimination from used endodontic files
\end{abstract}

Keywords: Biological debris, endodontic files, stereomicroscope

\section{Introduction}

According to guidelines of infection control cleaning of instruments to remove organic residue is a requisite to achieve sterility of instruments. ${ }^{1-4}$ Sterilization is a process that eliminates all forms of micro biota by physical/chemical methods. ${ }^{1}$ Disinfection is method that eliminates many or all pathogenic bacteria except spores. In health-care settings, objects usually are disinfected by liquid chemicals or wet pasteurization. ${ }^{1}$ Crossinfection is a major issue in the dental care setting because of risks about spread of disease via the oral cavity.

Endodontic treatment may directly involve contact with saliva, blood and infected pulp tissue. According to Australian National Health and Medical Research Council (NHMRC) guidelines (2002) ${ }^{1}$, instruments used in invasive dental procedures (including root canal treatment) are considered to involve a critical site, and should be sterile at the time of use. ${ }^{2}$ During the cleaning and shaping of the root canal, residual debris accumulates on the working sections of endodontic instruments. Transmission of these materials from one patient to another can cause extensive problems because they can act as antigens, infectious agents or nonspecific irritants. ${ }^{3}$ there has been exceptionally slight assessment of the effectiveness of cleaning procedures used for contaminated endodontic files.

Endodontic files and reamers do not have internal surfaces that are inaccessible, but their construction and designs, which involve fluted and twisted sections, make both mechanical and chemical cleaning considerably more complicated. Therefore, remaining biological debris may remain on the surface of the instrument even after sterilization. Thus, potentially infective material could be transmitted from an infected 
individual to other patients. Significant awareness has been directed to the possibility of transmission of prions via contaminated instruments. ${ }^{8}$

There is rising apprehension over the risk of iatrogenic spread of Creutzfeldt-Jakob disease (CJD). The repeated use of dental instruments presents a theoretical risk for the development of infection because previous studies have shown that the trigeminal ganglia, the periodontal, gingival and pulpal tissues, and the tonsils could represent a significant level of infectivity in patients with CJD. ${ }^{3}$

Thus, the need of the study was to evaluate which cleaning protocol has better effect on the elimination of biological debris and the level of contamination on the surfaces of used endodontic instruments so that occurrence of cross infection and CJD can be eliminated. As no study has been done on disinfection of used files using the criteria which are incorporated for the present study

Thus, the null hypothesis stated was that, "There will be no significant difference in the various cleaning protocols for eliminating debris and contamination of used endodontic instruments."

\section{Material And Method}

Ninety endodontic instruments (hand and rotary file systems), after clinical use in the Department of Conservative Dentistry and Endodontics, KMSDCH, Piparia, Vadodara were used for the study. Selection criteria were non corroded, used, contaminated, non deformed and unbroken hand and rotary instruments. The instruments were brushed manually with a nylon brush for 10 strokes and randomly divided into five groups depending on their cleaning protocols. Fifteen files directly from the manufacturer's packaging were used as control.

Group-1 ( $\mathrm{n}=15)$ Immersion in 3\% hydrogen peroxide for 10 minutes

Group-2 $(\mathrm{n}=15)$ immersion in $2 \%$ glutaraldehyde solution for 10 minutes

Group-3 $(\mathrm{n}=15)$ immersion in $0.2 \%$ chlorhexidine gluconate for 10 minutes.

Group-4 $(\mathrm{n}=15)$ immersion in $3 \%$ sodium hypochlorite for 10 minutes

Group -5 $(\mathrm{n}=15)$ ultrasonic bath with an enzymatic solution for 10 minutes.

All the instruments in the experimental groups were finally rinsed in distilled water for five minutes and dried.

Group-6 $(n=15)$ control group, the instruments were removed from their original packages, followed by rinsing in distilled water for 10 minutes and dried.( no nylon brushing strokes were given )

\subsection{Staining of endodontic hand instruments}

Once the cleaning procedure was done, all the instruments were stained using methylene blue stain for 3 minutes followed by rinsing in distilled water for five minutes and drying in an endodontic stand before visualizing under the stereomicroscope.

Special holder for placement of instruments under stereomicroscope: A holder was made of polyvinylsiloxane to provide a stable platform to view the instruments under the stereomicroscope. A small opening was made in the centre of holder to facilitate easy placement of instruments. The sides of the holder were marked as 1,2, 3 and 4 corresponding to the four sides. This arrangement helped in placing the instruments in proper position under stereomicroscope while being examined.

\subsection{Visualization under stereomicroscope:}

All the instruments were visualized for presence of residual organic debris at $40 \mathrm{X}$ magnification under stereomicroscope (MOTIC, DEPARTMENT OF ORAL PATHOLOGY, KMSDCH) and scoring was done.

Score 0: Clean surface,

Score 1: Organic film i e a thin, red unstructured layer covering a part of the instrument,

Score 2: Slight staining in the form of single particles,

Score 3: Moderate staining, organic particles covering the surface of instrument as continuous layer,

Score 4: High level of staining, with the cutting flutes completely covered with debris.

The instruments were examined by two different investigators in a blinding manner at three levels; apical, middle and coronal third. At each level the instruments was analyzed from four sides by sequential rotation of the special holder through 90 degrees, which gave 12 measurements for each instrument. Thus, the entire cutting surfaces of the instruments were examined. All the measurements were summed up. The minimum value is 0 (no organic debris material present; $0 \%$ ) and the maximum value would be 48 (all the surfaces contaminated strongly with organic debris; 100\%). The calculated value of each instrument was converted into percent value that was presented as the mean percentage of maximum biological contamination (MBC). The data obtained was tabulated and subjected to statistical analysis. 


\section{Results}

The data were then statistically analyzed by using Kruskal-Wallis Test and Mann Whitney U tests. A significant difference was observed in the amount of visible debris on instruments that had been cleaned in this study. Among experimental groups, highest percentage MBC values were found for instruments that were immersed in $2 \%$ glutaraldehyde The current study showed that even the unused files (group 6 - control group ) which were taken directly from the manufacturers packaging showed highest presence of stained and unstained debris on their instruments. Lowest percentage of MBC values for instruments was detected in the fifth group that were cleaned manually, chemically and by an ultrasound technique.

\section{Figures And Tables}

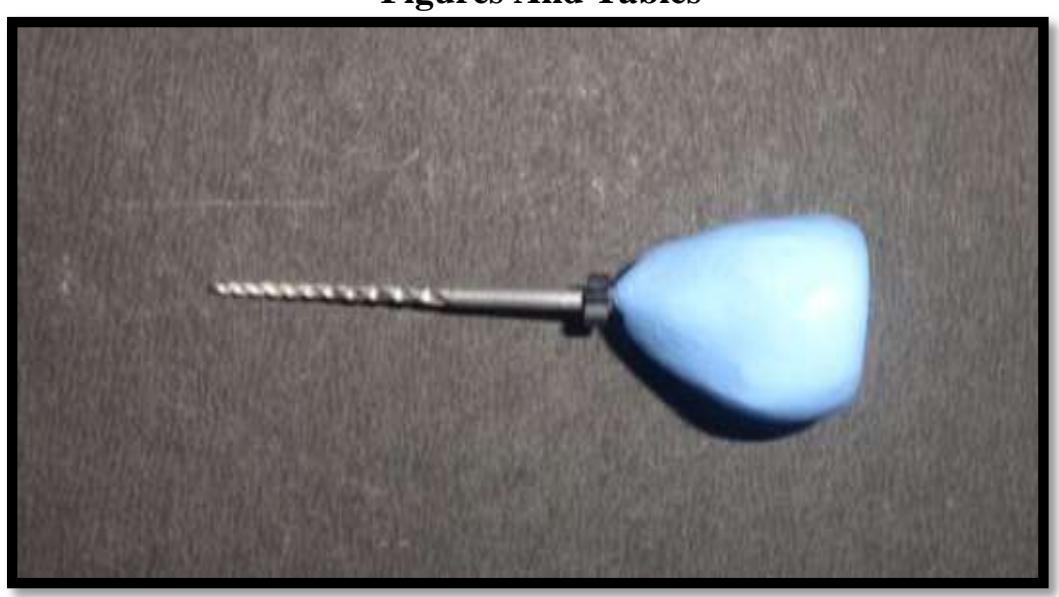

Figure 1

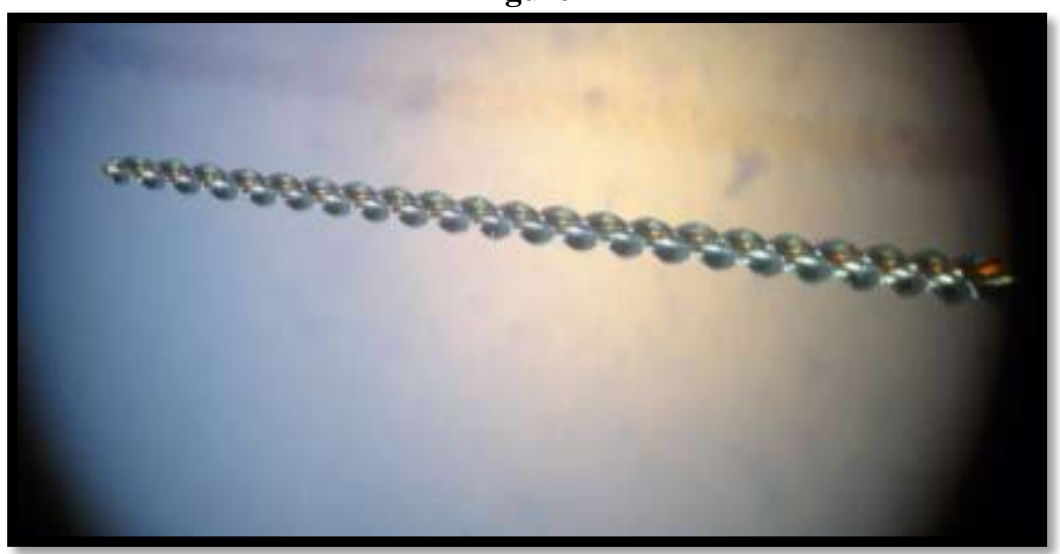

Figure 2: Group 1

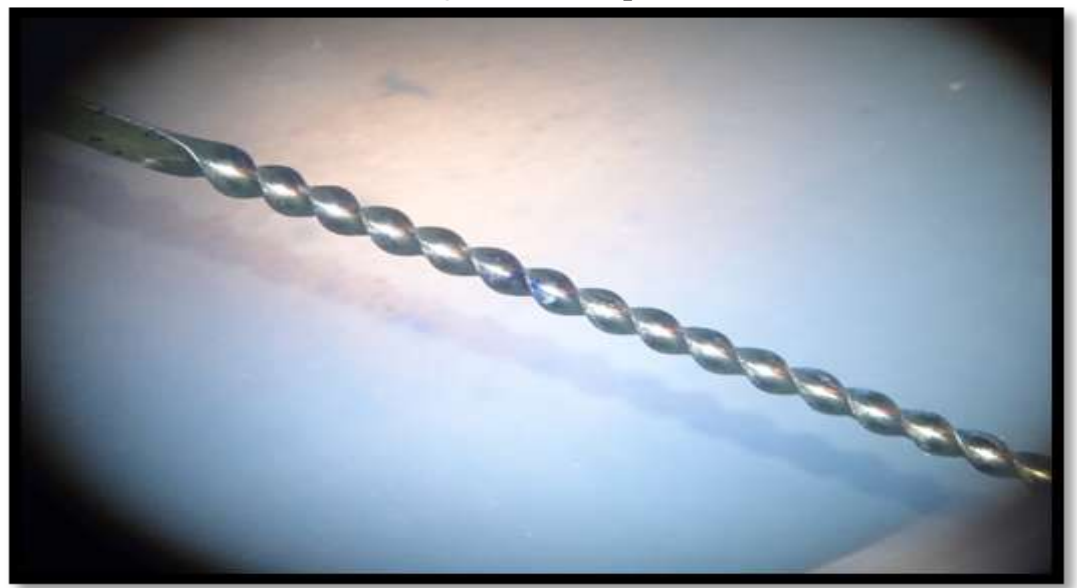

Figure 3: Group 2 


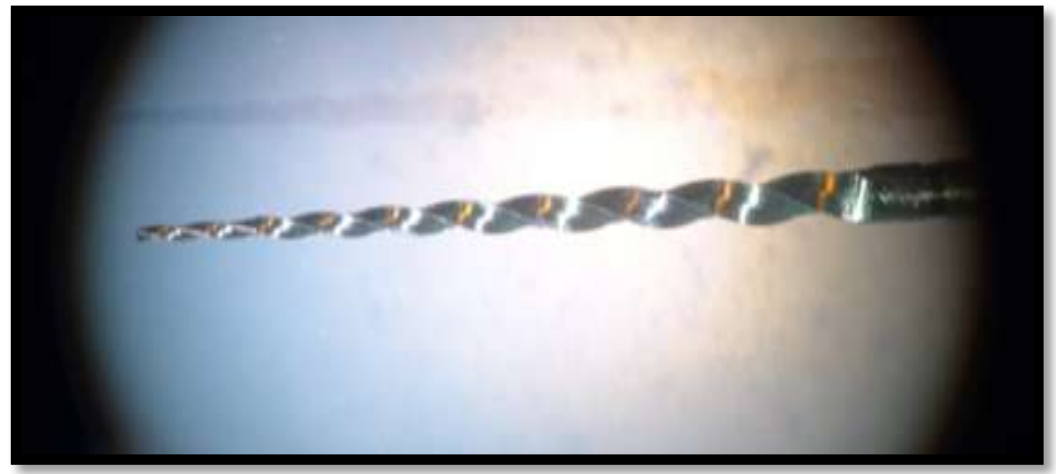

Figure 4: Group 3

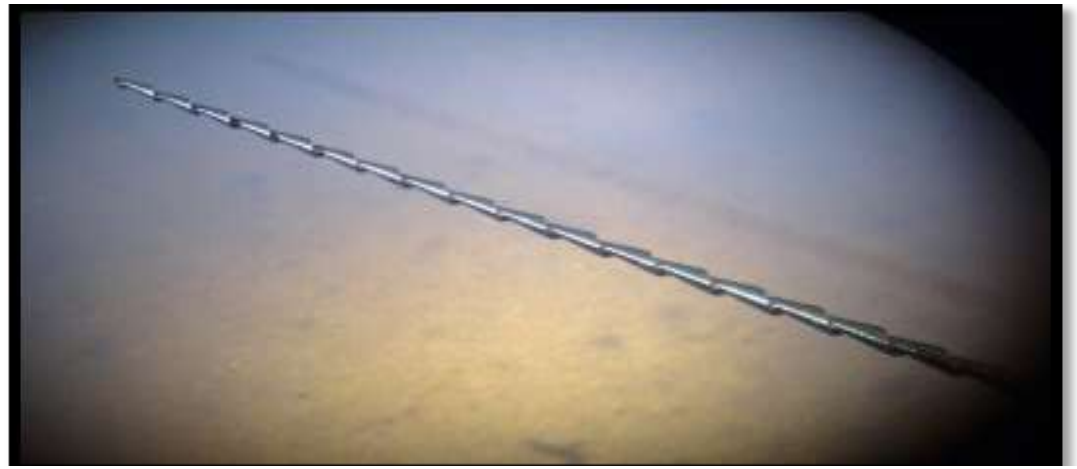

Figure 5: Group 4

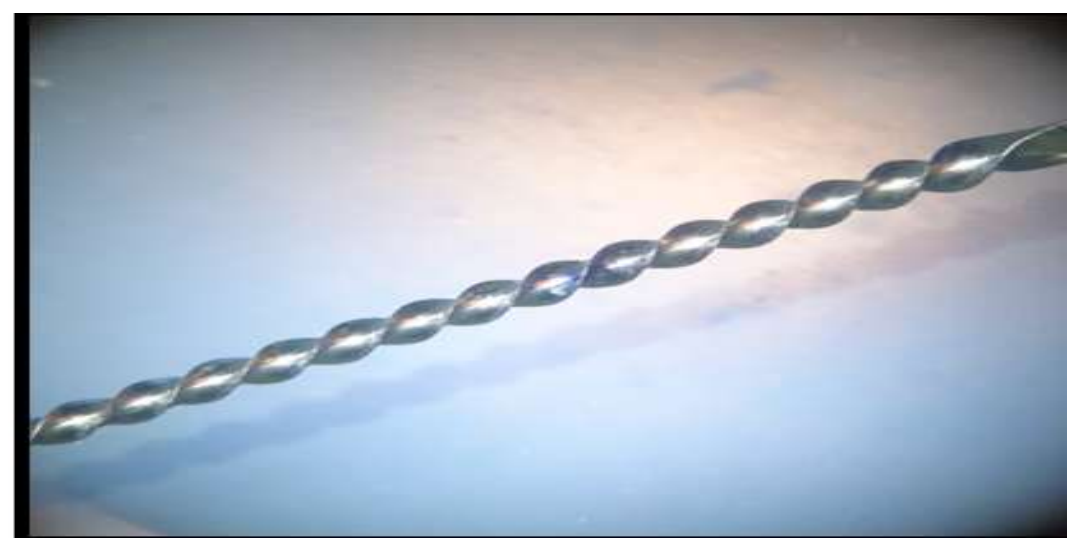

Figure 6: Group 5

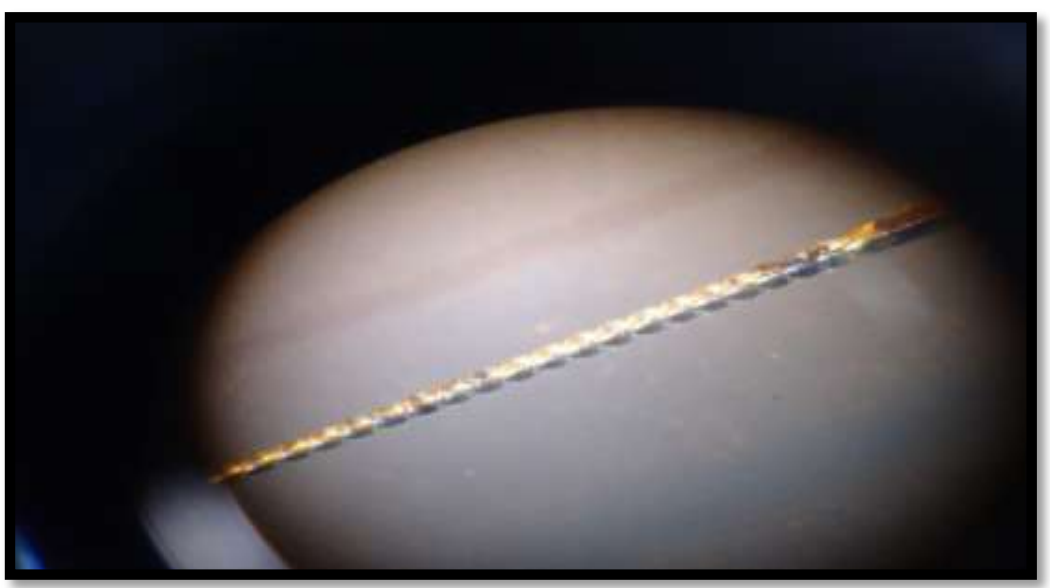

Figure 7: Group 6 
TABLE 1

\begin{tabular}{|ll|}
\hline Group $-\mathbf{1}(\mathbf{n}=\mathbf{1 5})$ & Immersion in 3\% hydrogen peroxide for 10 minutes \\
\hline Group $-\mathbf{2}(\mathbf{n}=\mathbf{1 5})$ & Immersion in 2\% glutaraldehyde solution for 10 minutes \\
\hline Group $-\mathbf{3}(\mathbf{n}=\mathbf{1 5})$ & immersion in $0.2 \%$ chlorhexidine gluconate for 10 minutes. \\
\hline Group $-\mathbf{4}(\mathbf{n}=\mathbf{1 5})$ & immersion in 3\% sodium hypochlorite for 10 minutes \\
\hline Group $-\mathbf{5}(\mathbf{n}=\mathbf{1 5})$ & ultrasonic bath with an enzymatic solution for 10 minutes. \\
\hline Group $-\mathbf{6}(\mathbf{n}=\mathbf{1 5})$ & unused instruments \\
\hline
\end{tabular}

TABLE 2

\begin{tabular}{|c|c|c|c|c|c|c|}
\hline CLEANING SCORE & GROUP 1 & GROUP 2 & GROUP 3 & GROUP 4 & GROUP 5 & GROUP 6 \\
\hline 4 & 0 & $5(33.33 \% 0$ & 0 & 0 & 0 & $6(40 \%)$ \\
\hline 3 & $1(6.66 \%)$ & $3(20 \%)$ & $2(13.33 \%)$ & $2(13.33 \%)$ & 0 & $5(33.33 \%)$ \\
\hline 2 & $3(20) \%$ & $4(26.66 \%)$ & $5(33.33 \%)$ & $5(33.33 \%)$ & $3(20 \%)$ & $4(26.66 \%)$ \\
\hline 1 & $10(66.66 \%)$ & $3(20 \%)$ & $7(46.66 \%)$ & $8(53.33 \%)$ & $8(53.33 \%)$ & 0 \\
\hline 0 & $1(6.66 \%)$ & 0 & $1(6.66 \%)$ & 0 & $4(26.66 \%)$ & 0 \\
\hline TOTAL & 15 & 15 & 15 & 15 & 15 & 15 \\
\hline
\end{tabular}

TABLE 3

\begin{tabular}{|l|l|}
\hline GROUP & Mean of MBC ( \%) \\
\hline 1- Immersion in 3\% hydrogen peroxide for 10 minutes & 2.63 \\
\hline 2 -Immersion in 2\% glutaraldehyde solution for 10 minutes & 5.69 \\
\hline 3 - Immersion in 0.2\% chlorhexidine gluconate for 10 minutes. & 3.19 \\
\hline 4 -Immersion in 3\% sodium hypochlorite for 10 minutes & 3.32 \\
\hline 5-Ultrasonic bath with an enzymatic solution for 10 minutes. & 1.94 \\
\hline 6- Unused instruments & 6.52 \\
\hline
\end{tabular}

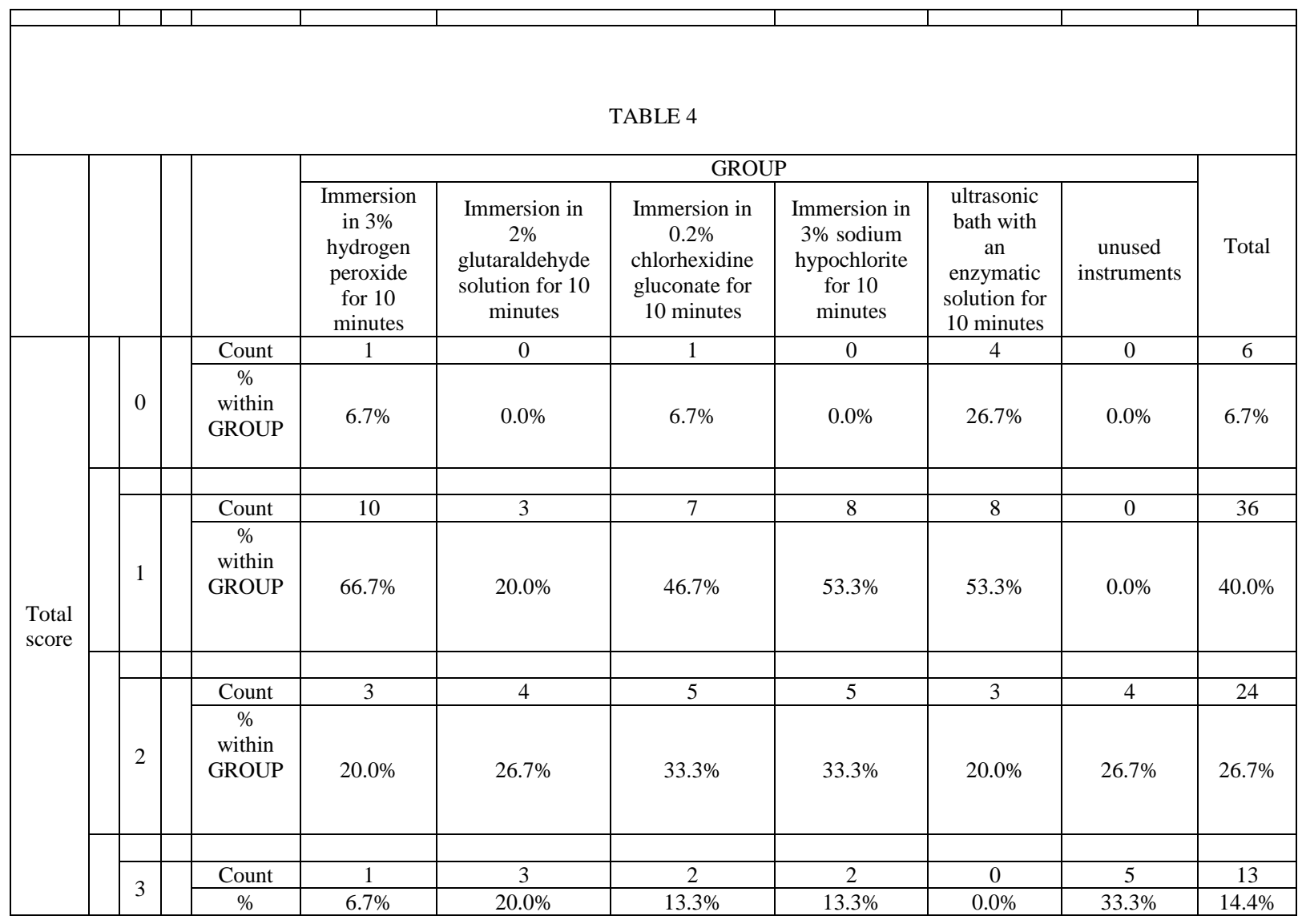


A Comparative Assessment Of Presence Of Biological Debris And Level Of Contamination ...

\begin{tabular}{|c|c|c|c|c|c|c|c|c|c|}
\hline & & $\begin{array}{l}\text { within } \\
\text { GROUP }\end{array}$ & & & & & & & \\
\hline & & Count & 0 & 4 & 0 & 0 & 0 & 6 & 10 \\
\hline & 4 & $\begin{array}{c}\% \\
\text { within } \\
\text { GROUP }\end{array}$ & $0.0 \%$ & $26.7 \%$ & $0.0 \%$ & $0.0 \%$ & $0.0 \%$ & $40.0 \%$ & $11.1 \%$ \\
\hline & & Count & 0 & 1 & 0 & 0 & 0 & 0 & 1 \\
\hline & 5 & $\begin{array}{c}\% \\
\text { within } \\
\text { GROUP }\end{array}$ & $0.0 \%$ & $6.7 \%$ & $0.0 \%$ & $0.0 \%$ & $0.0 \%$ & $0.0 \%$ & $1.1 \%$ \\
\hline & & & & & & & & & \\
\hline \multirow[b]{2}{*}{ Total } & & Count & 15 & 15 & 15 & 15 & 15 & 15 & 90 \\
\hline & & $\begin{array}{c}\% \\
\text { within } \\
\text { GROUP }\end{array}$ & $100.0 \%$ & $100.0 \%$ & $100.0 \%$ & $100.0 \%$ & $100.0 \%$ & $100.0 \%$ & $100.0 \%$ \\
\hline
\end{tabular}

TABLE 5 :KRUSKAL -WALLIS TEST

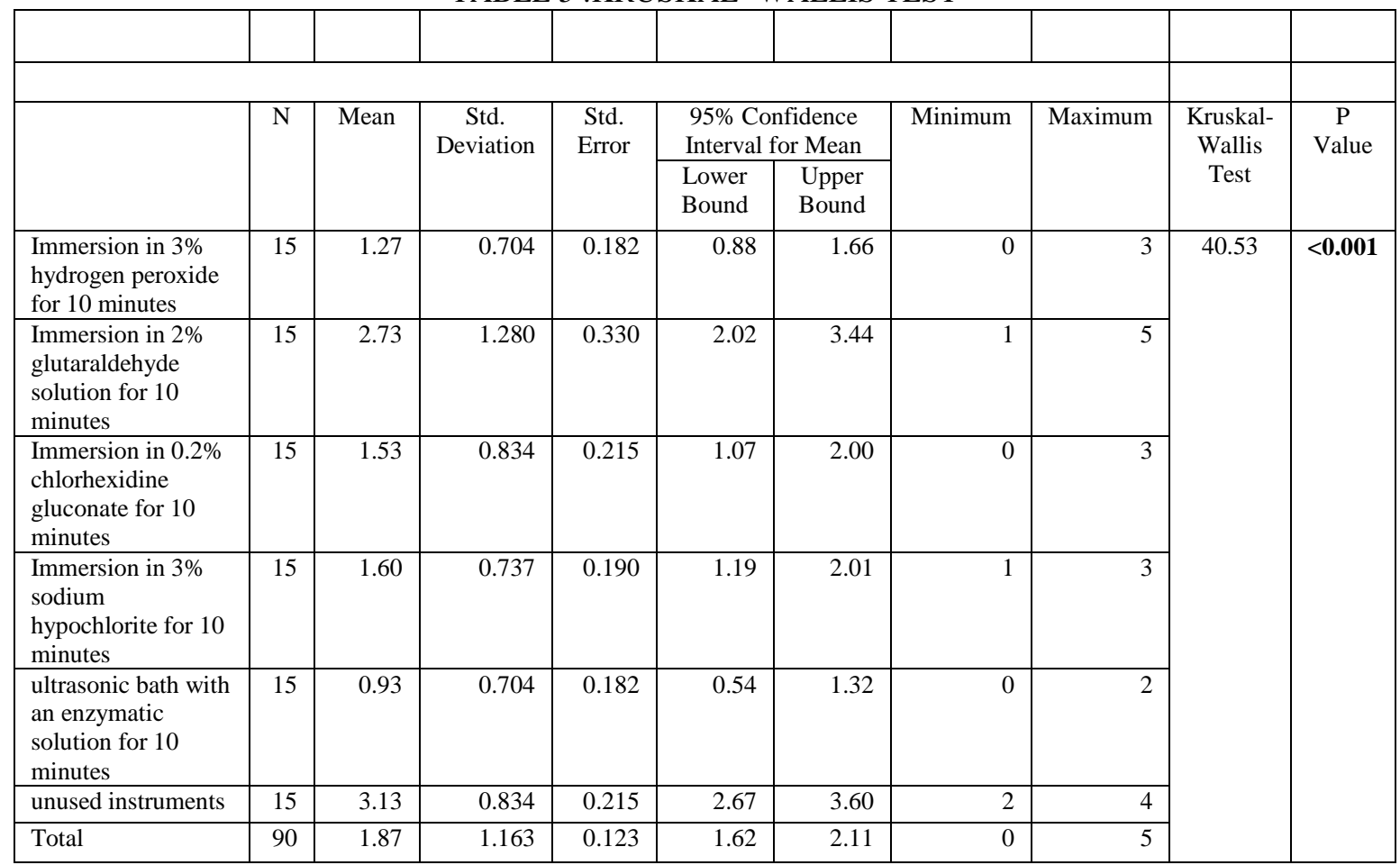


Table 6

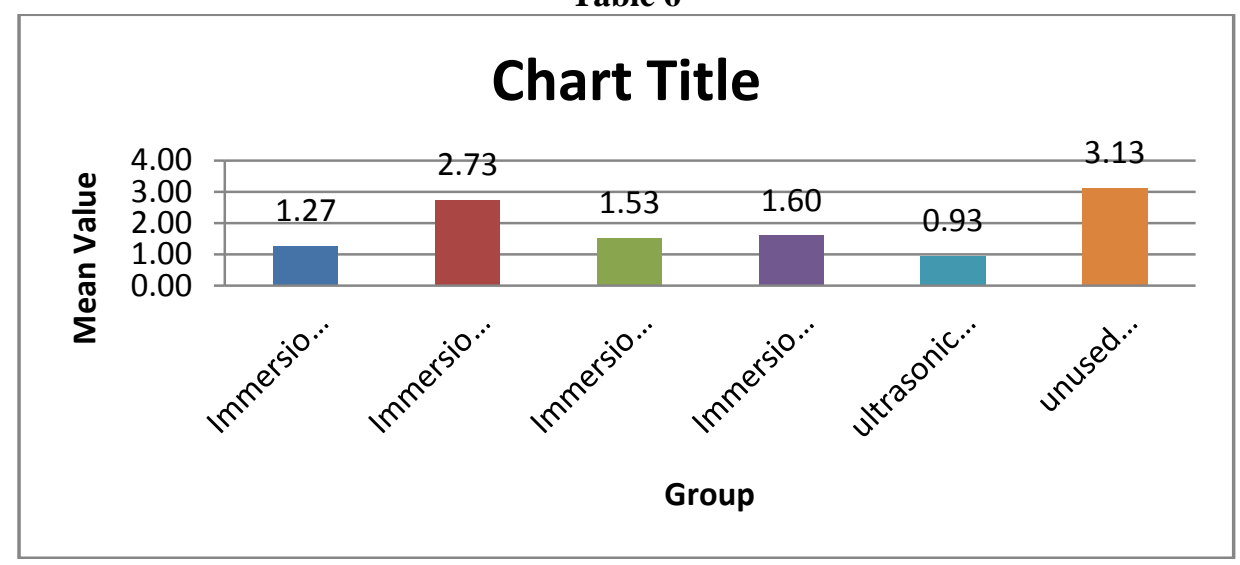

\section{Discussion}

Pre-cleaning and sterilization of some instruments can be difficult because of their complex design.Cleaning and shaping of root canal system is an important phase of endodontic treatment. This is usually performed using hand and rotary instruments which are often reused. As root canal instrumentation renders gathering of debris on the flutes of the file, these instruments have to be cleaned, disinfected and sterilized effectively. Residual debris may remain adhered to the surface of endodontic instruments even after standard procedures of cleaning and sterilization. One reason for this is the complex design of the files, which have fluted and twisted sections making mechanical and chemical cleaning considerably more difficult. This residual debris can prevent the disinfectant from contacting the instrument; it may bind and inactivate the chemical disinfectants thus interfering with the sterilization. In addition there is a risk of various bacterial, viral and the prion diseases such as a variant of a Creutzfeldt-Jakob disease (v CJD). ${ }^{(1,4,18)}$

In spite of several methods available for cleaning there is little consistent information on the optimum method of cleaning. Hence the present study was undertaken to appraise the efficacy of several cleaning protocols on the elimination of biological debris and the altitude of contamination on used endodontic instruments that were subjected to different cleaning protocols using a stereomicroscope.

In the current study, used endodontic files collected from Department of Conservative Dentistry \& Endodontics, KMSDCH were selected to determine one pre sterilization protocol for biological debris elimination in day to day clinical practice. As several literatures has stated that along with chemical soaking and cleaning, manual brushing is equally important for removal of debris from the used endodontic files.Initial cleaning with a brush is important as it removes considerable amount of debris and it is simple and quick to perform for the clinician and the assistant. Syed Ziauudin et al (2013) ${ }^{42}$, who used nylon brushing (20 strokes) and $2 \times 2$ sized gauze for manual cleaning prior to any chemical or mechanical methods. Thus, in this study the manual cleaning method - nylon brushing (10 strokes) was done for all the specimens prior to any chemical /mechanical cleaning and various chemicals used were 3\% hydrogen peroxide, $2 \%$ glutardehyde, $0.2 \%$ chlorhexidine gluconate $3 \%$ sodium hypochlorite and an enzymatic solution with ultrasonic agitation.

Hydrogen Peroxide (3\%) tested in this study, produces transient yet energetic effervescence that displaces debris. The bubbling action of the solution when in contact with tissues physically foams debris out of the instruments. It acts on the microorganisms through its release of nascent oxygen. Hydrogen peroxide is used in hospitals to disinfect surfaces. Breakdown in light, decomposition by catalase and lessening of activity by organic matter is its disadvantages (Favero\&Bond, 1991).

2\% Glutaraldehyde,(Gravenmade \& Dankert. 1975) was used in this study, as it is a strong disinfectant, fixative and diminishes microbes by changing the essential protein compounds. ${ }^{18}$ They have a wide microbiocidal activity and are sporocidal and fungicidal (Crawford, 1983).Disadvantages of these agents are: Vapors are irritating and must be neutralized by ammonia, have poor dispersion, leave non-volatile remains, action is reduced in the presence of protein. Some bacteria have developed resistance to glutaraldehyde). ${ }^{4}$

$0.2 \%$ Chlorhexidine gluconate is also used for pre sterilization procedure in this study as it is a strong base and is most stable in the form of its salts. It has a cationic molecular component that attaches to negatively charged cell membrane area and causes cell lysis. Chlorhexidine is a potent antiseptic and has high substantivity.

Sodium hypochlorite (3\%) which has been previously tested for its cleaning effectiveness was also included in this study. Besides their wide-spectrum, nonspecific killing efficacy on all microbes, hypochlorite preparations are sporocidal, virucidal, and greater tissue dissolving effect on necrotic tissues (Austin \& Taylor, 
1918).The antibacterial effectiveness and tissue-dissolution capacity of aqueous hypochlorite is a function of its concentration, but so is its toxicity (Spyngbergl et al., 1973).

Enzymatic detergents (Bio Sonic) such as the one used in this study are at present extensively suggested for the cleaning of medical devices as they remove proteins, lipids and carbohydrates from the instrument plane. It is a faster and easier procedure for cleaning dental instruments ${ }^{3,8}$. Cavitation activity of the ultrasonic bath along with chemical activity of the detergent helps to remove biologic debris ${ }^{3}$.

In previous studies Van Gieson's staining method was mainly used to demonstrate organic debris. Van Gieson's solution is a mixture of picric acid and acid fuchsin where as in present study methylene blue dye was used because it is simple, readily available and renders contrast color to biological debris and makes it more detectable.

In the current study, for debris evaluation stereomicroscope was used because the stereo or stereoscopic or dissecting microscope is an optical microscope variation intended for low magnification observation of a sample, typically using reflected light than the transmitted one. This arrangement produces a three-dimensional visualization of the sample being examined.

A significant difference was observed in the amount of visible debris on instruments that had been cleaned in this study. This may be the consequence of poor performance of the cleaning protocol in practice. Poor cleaning occurred particularly in $2 \%$ glutaraldehyde solution for 10 minutes (Group 2) and unused instruments rinsed with distilled water without any manual brushing (Group $6-$ control group ). Among experimental groups, highest percentage MBC values were found for instruments that were immersed in $2 \%$ glutaraldehyde This could be because glutarldehyde being a bactericidal, fixative does not loosens the adherent debris and the time of immersion is only 10 minutes. The current study showed that even the unused files (group 6 - control group ) which were taken directly from the manufacturers packaging showed highest presence of stained and unstained debris on their instruments. This is in agreement with the previous studies done by Sonntag \& Peters et al (2007) ${ }^{32}$.

Lowest percentage of MBC values for instruments was detected in the fifth group that were cleaned manually, chemically and by an ultrasound technique. This could be attributed to the effectiveness of ultrasonic waves in combination with manual cleaning with the brush and an efficient detergent. The present study has shown that the use of ultrasonic along with enzymatic detergent is an important step in instrument cleansing and this is in agreement with previous studies done by Popovic (2010) ${ }^{2}$, Tanomaru Filho(2001) ${ }^{16}$ and Qualtrough $(2006)^{31}$ et al .

Thus, null hypothesis was rejected as groups are showing statistically significance difference in cleaning ability of used endodontic files.

\section{Conclusion}

From the results of our study it can be concluded that pre cleaning of endodontic files is simple method $\&$ can be easily learned and implemented in private practice or institution.

\section{References}

[1]. Guideline for Disinfection and Sterilization in Healthcare Facilities, 2008

[2]. Popovic J, Gasic J, Zivkovic S, Petrovic A, Radicevic G. Evaluation of biological debris on endodontic instruments after cleaning and sterilization procedures. Int Endod Journal 2010; 43: 336-341.

[3]. Segall RO, del Rio CE, Brady JM, Ayer WA. Evaluation of debridement techniques for endodontic instruments. Oral Surg Oral Med Oral Pathol 1977; 44: 786-791.

[4]. Murgel CAF, Walton RE, Rittman B, Pecora JD. A comparison of techniques for cleaning endodontic files after usage: a quantitative scanning electron microscopic study. J Endod 1990; 16: 214-217.

[5]. Smith AJ, Dickson M, Aitken J, Bagg J. Contaminated dental instruments. J Hosp Infect 2002; 51: $233-235$.

[6]. Letters S, Smith AJ, McHugh S, Bagg J. A study of visual and blood contamination onreprocessed endodontic files from general dental practice. British Dental Journal 2005; 199: 522- 525.

[7]. Joint AAE/CAE Special Committee on Single Use Endodontic Instruments (Dr. Gary Hartwell - Chair, Dr. Walter Bowles, Dr. Ove Peters, Dr. Marshall Peikoff and Dr. Calvin Torneck): American Association of Endodontics.

[8]. Muqbil I, Burke FJT, Miller CH, Palenik CJ. Antimicrobial activity of ultrasonic cleaners. Journal of Hospital Infection 2005; 60: 249-255.

[9]. Raquel Assed Bezerra da Silva, Mário Roberto Leonardo, Lúcia Helena Faccioli, Alexandra Ivo Medeiros,Paulo Nelson- Filho. Effect of different methods of sterilization on the inactivation of bacterial endotoxin (LPS) in endodontic files. Brazilian Journal of Microbiology 2007;38:270-272.

[10]. S. A. Aasim, A. C. Mellor, A. J. E. Qualtrough. The effect of pre-soaking and time in the ultrasonic cleaner on the cleanliness of sterilized endodontic Files. International Endodontic Journal 2006;39:143-149.

[11]. DA Van Eldik, PS Zilm, AH Rogers, PD Marin. Microbiological evaluation of endodontic files after cleaning and steam sterilization procedures. Australian Dental Journal 2004;49:(3):122-127.

[12]. P Parashos, P Linsuwanont, HH Messer. A cleaning protocol for rotary nickel-titanium endodontic instruments. Australian Dental Journal 2004;49:(1):20-27.

[13]. Archie Morrison, Susan Conrod. Dental Burs and Endodontic Files:Are Routine Sterilization Procedures Effective? jcda 2009;.75(1):39a-39d. 
[14]. Boyd KS, Sonntag KD, Crawford JJ. Efficacy of sterilization of endodontic files after autoclaving in a synthetic sponge. Int Endod J. 1994;27(6):330-333.

[15]. Mitchell BF, James GA, Nelson RC. The effect of autoclave sterilization on endodontic files. Oral Surg Oral Med Oral Pathol. 1983;55(2):204-207.

[16]. M,Tanomaru Filho, M.R.Leonardo, K.C.Bonifacio, F.R.Dametto. The use of ultrasound for cleaning the surface of stainless steel and NiTi endodontic instruments. Int Endod J,2001;34:581-85.

[17]. P.Linasuwanont, P.Parashos, H H Messer. Cleaning of rotary nickel-titanium endodontic instruments., Int Endod J 2004,;37:19-28.

[18]. P Parashos, P Linsuwanont, HH Messer. Effective cleaning protocols for rotary nickel-titanium files. Australian Endodontic Journal,2003;29;23-4.

[19]. Hubbard Jr. TM, Robert N. Smyth, Pellen Jr., Joseph I.Tenca. Chair side decontamination of endodontic files. Oral Surg 1975;40(1):148-52. 